\title{
Optimal control and construction design of variable-geometry suspension systems
}

\author{
B. Németh and P. Gáspár \\ Systems and Control Laboratory, MTA SZTAKI Institute for Computer Science and Control, Hungarian \\ Academy of Sciences, Kende u. 13-17, H-1111 Budapest, Hungary
}

\begin{abstract}
The paper proposes methods for the analysis and the control design of variablegeometry suspension systems. The tilting actuation of the front wheel improves the lateral dynamics of the vehicle and assists the driver in avoiding critical situations. The novelty of the method is the consideration of the nonlinearities in the tyre characteristics. The nonlinear polynomial Sum-of-Squares (SOS) programming method is applied in the analysis and it gives the optimal utilization of the maximum control forces on the tires. Moreover, the construction of the system can be based on the nonlinear analysis. An LPV (Linear Parameter-Varying) based control-oriented modeling and control design for lateral vehicle dynamics are also proposed, which guarantee the trajectory tracking of the vehicle. The simulation example presents the efficiency of the variable-geometry suspension system and it shows that the system is suitable to be used as a driver assistance system.
\end{abstract}

\section{INTRODUCTION}

The variable-geometry suspension system is a mechanism with which road holding can be improved. The illustration of the system is found in Figure 1(a). The actuation of the control system is the lateral motion of point $A$, which can be generated by electro-hydraulic (Lee et al. 2006) or electric power (Evers et al. 2008). The motion affects the tilting of the wheel, by which the lateral dynamics of the vehicle is influenced. Moreover, the suspension determines such components as the height of the roll center and the half-track change. The roll center can be modified by setting the camber angle of the wheels. Thus, during maneuvers the control system must guarantee various crucial vehicle performances such as trajectory tracking, roll stability and geometry limits. The advantages of the mechanism are the simple structure, low energy consumption and low cost compared to other mechatronic solutions.

Several papers for various kinematic models of suspension systems have already been published, see e.g. Fallah et al. 2009, Németh \& Gáspár 2012b. The kinematic design of a doublewishbone suspension system, the variable height of the roll center and the half-track change have been in the focus of research. The vehicle-handling characteristics based on a variable roll center suspension were proposed by Lee et al. 2008. A rear-suspension active toe control for the enhancement of driving stability was proposed by Goodarzia et al. 2010. Another field of variablegeometry suspension is the steering of narrow vehicles, see Piyabongkarn et al. 2004. These vehicles require the design of an innovative active wheel tilt and steering control strategies in order to perform steering similarly to a car on straight roads but in bends they tilt as motorcycles, see Suarez 2012. The preliminary results of the set-based analysis were proposed in Németh \& Gáspár, 2012a.

In the design of variable-geometry suspension several factors must be considered. First, the construction of the system significantly determines the characteristics of the suspension, e.g. the effect of the actuation $a_{y}$ on the variation of camber angle $\gamma$. Second, in the control design method the required performances of the closed-loop system are formulated. An example of this relation is presented below. The modification of the lateral position of $A$, denoted by $a_{y}$, affects the rotation of the front wheel around axis $B K$, see Figure 1(b). Thus, the camber angle $\gamma$ and the 
steering angle $\delta_{c}$ are simultaneously changed. The position of the steering track-rod connection point $K$ has an important role in the distribution of $\delta_{c}$ and $\gamma$. Thus, it is necessary to choose a vertical position $K_{z}$, by which the lateral force on the tire is improved effectively. In the analyzed construction $K_{z}$ has a significant influence on $\delta_{c}$ and with an increased $K_{z}$ it is possible to achieve high lateral tire force. Moreover, $K_{z}$ influences the lateral movement of $T$, i.e., the half-track change, which is denoted by $\Delta B$. It has an important role in tire wear. Consequently, the steering angle, the camber angle and the half-track change are functions of the actuation. Since the steering and the tilting angles determine the lateral force on the wheel, the balance between these signals is significant in the design of the vehicle dynamic controller.

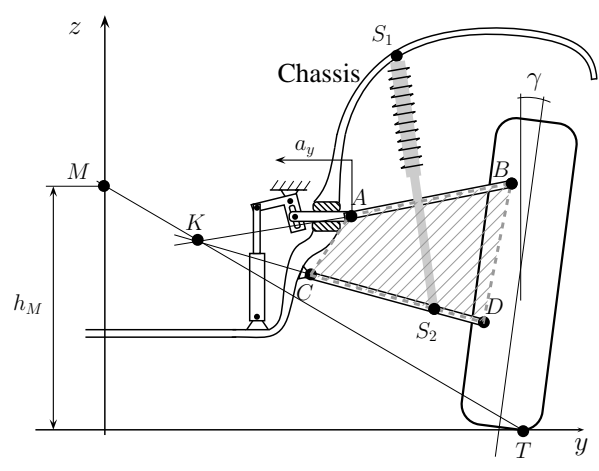

(a) Variable-geometry suspension system

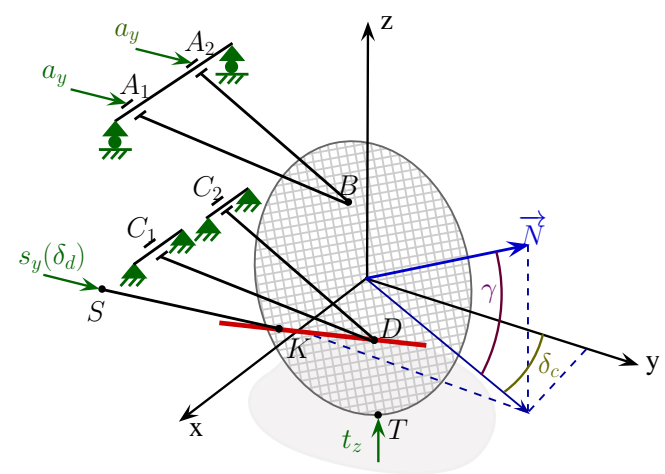

(b) Wheel position related to the steering and the camber angle

Figure 1. Modeling of variable-geometry suspension construction

In the following parts of the paper the modeling and stability analysis of the suspension system based on set theoretic methods are briefly presented. Moreover, an LPV-based control design method is proposed with vehicle dynamic simulations. Finally, the most important contributions of the paper are summarized.

\section{ANALYSIS OF THE SYSTEM BASED ON INVARIANT SETS}

In the paper the lateral forces of the tire are approximated with polynomial functions. Although several methods for the modeling of the tire have already been published, see e.g., Pacejka 2004, Kiencke \& Nielsen 2000, de Wit et al. 1995, the polynomial modeling has three main advantages in the stability analysis. First, the lateral forces of the tire in an extended nonlinear region can be included. Second, the mathematical model of the tire model is compact, which results in a relatively easy application in vehicle modeling. Third, the polynomial formulation makes it possible to use the systematic Sum-of-Squares (SOS) method for the analysis. The disadvantage of the polynomial approximation is the numerical complexity of the polynomials. Improving the accuracy of the tire force characteristics results in an increase in the degree of polynomials.

In the case of the variable-geometry suspension system two nonlinearities of the tire characteristics must be considered in a given operation range. Lateral tire force $\mathcal{F}(\alpha)$ depends on the lateral tire slip $\alpha$ nonlinearly. Although in several control applications the lateral forces are approximated with linear functions, which results in a simple description, the lateral tire forces can be used in a narrow tire side-slip range. Vehicle motion is significantly characterized by this nonlinearity. The generated lateral tire force from camber angle $\mathcal{G}(\alpha)$ depends on $\alpha$ nonlinearly. Thus, the efficiency of actuator intervention is influenced by the tire slip.

The nonlinear model of the tire is constructed from the polynomial approximation of the previous two effects, $\mathcal{F}(\alpha)$ and $\mathcal{G}(\alpha)$, as $\mathbf{F}_{\text {lat }}(\alpha)=\mathcal{F}(\alpha)+\mathcal{G}(\alpha) \gamma=\sum_{j=1}^{n} c_{j} \alpha^{j}+\sum_{k=0}^{m} g_{k} \alpha^{k} \gamma$. 


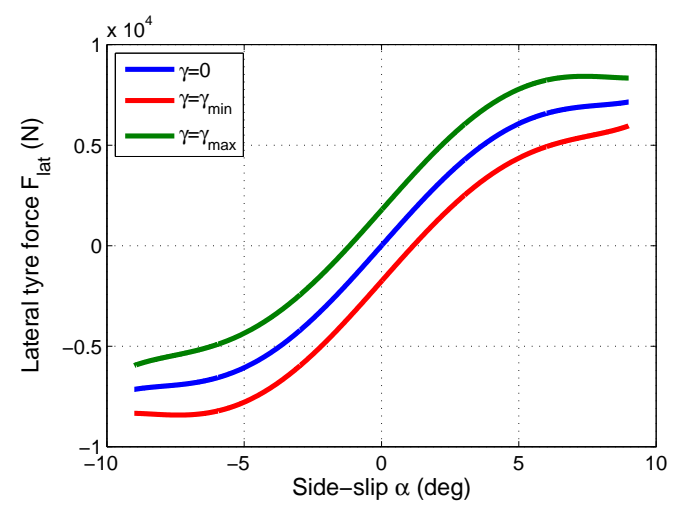

Figure 2. Modeling of lateral tire force $\mathbf{F}_{\text {lat }}$

The lateral model of the vehicle, which describes the lateral forces and the torques in the lateral plane, is formulated by Németh \& Gáspár 2014:

$\dot{x}=\left[\begin{array}{c}\dot{\alpha}_{1} \\ \dot{\alpha}_{2}\end{array}\right]=\left[\begin{array}{l}\mathrm{f}_{1}\left(\alpha_{1}, \alpha_{2}\right) \\ \mathrm{f}_{2}\left(\alpha_{1}, \alpha_{2}\right)\end{array}\right]+\left[\begin{array}{ll}\mathrm{h}_{11} & \mathrm{~h}_{12} \mathcal{G}\left(\alpha_{1}\right) \\ \mathrm{h}_{21} & \mathrm{~h}_{11} \mathcal{G}\left(\alpha_{1}\right)\end{array}\right]\left[\begin{array}{l}\delta \\ \gamma\end{array}\right]$

where the state vector $x=\left[\begin{array}{ll}\alpha_{1} & \alpha_{2}\end{array}\right]^{T}$ contains the lateral slips on the front and rear wheels and $\mathrm{f}_{i}, \mathrm{~h}_{i j}$ are parameters.

The stability analysis of the variable-geometry suspension system is based on the Maximum Controlled Invariant Sets. In the method the states of the system are found, in which the stability of the system considering the peak-bounded control input limits can be guaranteed. The application details of the SOS programming in the set computation are found in Németh \& Gáspár 2014. In the following the results of the set computation for a variable-geometry suspension of a lightweight vehicle are proposed. Figure 3(a) shows the results with the parameters $K_{z}=300 \mathrm{~mm},\left|\delta_{c, \max }\right|=18^{\circ}$ compared to an individual steering actuation. It can be seen, that the sets, where the suspension is able to stabilize the system, are larger than the sets of the steering actuation. The distance of the set boundary from zero increases by approximately $10 \%$. The increase is resulted from the joint actuation of steering and tilting. Furthermore the analysis illustrates that the increase in the velocity reduces the sets. In the background of this effect the increase in the unstable sets at higher velocity is found. In Figure 3(b) the Maximum Controlled Invariant

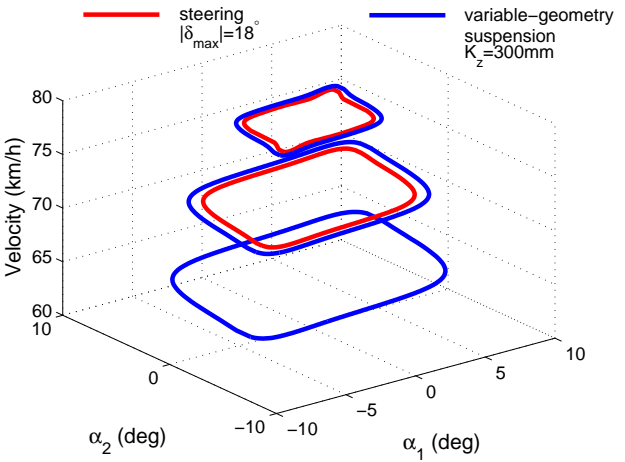

(a) Sets of the systems at different velocities

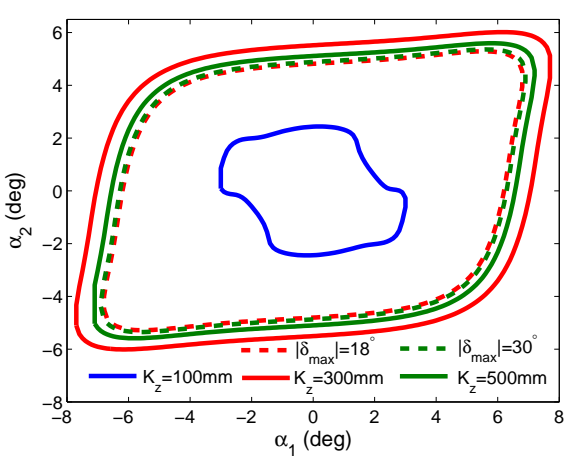

(b) Sets of the systems at fixed velocity $v=$ $72 \mathrm{~km} / \mathrm{h}$

Figure 3. Maximum Controlled Invariant Sets of the systems

Sets of different $K_{z}$ values are compared at a fixed velocity. When $K_{z, 1}=100 \mathrm{~mm}$ the wheel tilting intervention is dominant, while steering has a slight counter-influence. $K_{z, 2}=300 \mathrm{~mm}$ 
leads to a balance between the camber and the steering angle, while at $K_{z, 3}=500 \mathrm{~mm}$ steering is preferred. Moreover, construction parameter $K_{z, 1}$ results in a small region at both velocities. Thus, camber angle intervention is insufficient by itself, so it is necessary to find a $K_{z}$, which affects $\gamma$ and $\delta_{c}$ angles simultaneously.

It can be seen that the simultaneous actuation of steering and wheel tilting leads to the enlargement of $\mathbf{S}$, see $K_{z, 2}$ and $K_{z, 3}$ scenarios. However, the enlargement is more significant at $K_{z, 2}$. Moreover, the $\mathbf{S}$ region of $K_{z, 2}$ is larger than the region of $K_{z, 3}$. The reason for these phenomena is the reduction of the maximum $\gamma$ at high $K_{z}$ values.

\section{CONTROL DESIGN BASED ON THE VARIABLE-GEOMETRY SUSPENSION}

The primary role of the variable-geometry suspension system is to support the driver activities, i.e., it is a driver assistant control system. In the trajectory tracking control the lateral dynamics must be taken into consideration and the vehicle must follow the reference yaw-rate signal, which is generated by the driver steering angle $\delta_{d}$. The other performances are related to the dynamic displacement of the roll center, the half track change and the control input $a_{y}$.

In the following the polynomial description of the tyre model is transformed into a controloriented form. The linearizing of $\mathbf{F}_{\text {lat }}(\alpha)$ around a given $\alpha_{0}$ leads to the following expression:

$$
\left.F(\alpha)\right|_{\alpha_{0}}=F_{0}\left(\alpha_{0}\right)+c\left(\alpha_{0}\right) \alpha+G_{0}\left(\alpha_{0}\right) \gamma
$$

where $c\left(\alpha_{0}\right)$ is cornering stiffness. In (2) the parameters $F_{0}\left(\alpha_{0}\right)$ and $c\left(\alpha_{0}\right)$ depend on the slip value $\alpha_{0}$ in the tire model. $F_{0}\left(\alpha_{0}\right)=\mathcal{F}\left(\alpha_{0}\right)$ and $G_{0}\left(\alpha_{0}\right)=\mathcal{G}\left(\alpha_{0}\right)$ are the values of the lateral tire force at $\alpha_{0}$, while $c\left(\alpha_{0}\right)=\left.\frac{d \mathcal{F}(\alpha)}{d \alpha}\right|_{\alpha_{0}}$ represents the linear slope at $\alpha_{0}$. In this way the polynomial vehicle model (1) is transformed into an LPV form:

$\dot{x}=A\left(\rho_{1}, \rho_{2}\right) x+B_{1}\left(\rho_{1}, \rho_{2}\right) w+B_{2}\left(\rho_{1}, \rho_{2}\right) u+W\left(\rho_{1}, \rho_{2}\right)$,

where $x=\left[\begin{array}{ll}\dot{\psi} & \beta\end{array}\right]^{T}$ is the state vector, the control input is the lateral motion $u=a_{y}, \rho_{1}=\alpha_{0,1}$ and $\rho_{2}=\alpha_{0,2}$ scheduling variables are the lateral slips of the front/rear axles.

The main performance specification of the control system is trajectory tracking. In trajectory tracking control the vehicle must follow the reference yaw rate, i.e., the purpose is to minimize the difference between the current yaw rate of the vehicle and the reference yaw rate:

$z=\left|\dot{\psi}_{\text {ref }}-\dot{\psi}\right| \rightarrow \min$

The reference signal is generated by the driver himself in a driver assistance system or it can be computed from the curvature of the road.

The goal of the control system is to guarantee performance (4) by an appropriate control input $u$. The designed LPV control computes this signal based on the measured tracking error. In the LPV control design several weighting functions are used, which scale the gain and the frequency range of the signals. The role of the weighting function $W_{r e f}$ is to scale $\dot{\psi}_{\text {ref }}$. The weighting function $W_{w}$ scales disturbance $W$, while $W_{e}$ scales the sensor noise on the measurement. The tracking performance of the system is guaranteed by the weighting function $W_{p}$. The closed-loop architecture of the system is illustrated in Figure 4.

The quadratic LPV performance problem is to choose the parameter-varying controller $K\left(\rho_{1}, \rho_{2}\right)$ in such a way that the resulting closed-loop system is quadratically stable and the induced $\mathcal{L}_{2}$ norm from the disturbance $w$ and the performances $z$ is less than the value $\Gamma$, see Bokor \& Balas 2005 . The minimization task is the following:

$\inf _{K} \sup _{\varrho \in \mathcal{F}_{\mathcal{P}}} \sup _{\|w\|_{2} \neq 0, w \in \mathcal{L}_{2}} \frac{\|z\|_{2}}{\|w\|_{2}}$.

The existence of a controller that solves the quadratic LPV $\Gamma$-performance problem can be expressed as the feasibility of a set of Linear Matrix Inequalities (LMIs), which can be solved numerically. Finally, the state space representation of the LPV control $K\left(\rho_{1}, \rho_{2}\right)$ is constructed, see $\mathrm{Wu}$ et al. 1996. 


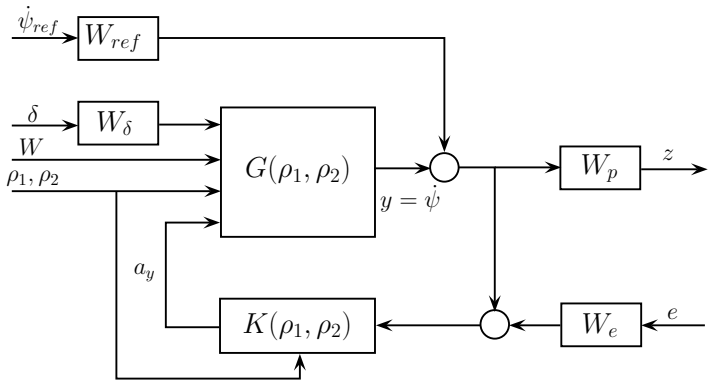

Figure 4. Closed-loop interconnection

\section{SIMULATION RESULTS}

The efficiency of the method is presented using a simulation scenario. The vehicle must perform a double lane change maneuver with a constant velocity $v=130 \mathrm{~km} / \mathrm{h}$. The vehicle must remain within the lanes. The purpose of the controller is the design of the appropriate actuation $a_{y}$, which generates front-wheel camber angle $\gamma$.

In the simulation two scenarios are compared. In the first case the vehicle is controlled by the proposed LPV controller. In the second case the vehicle is uncontrolled, driver intervention is performed. In a double lane change test usually the final section is critical, when the vehicle reaches one of the borders. Since the purpose of the control is to keep the lanes, the end of the path is a good reference for checking controller efficiency.

Figure 5(a) illustrates the motions of the two vehicles, the LPV controlled and the uncontrolled vehicle. It can be stated that there are two critical points along the lane where the uncontrolled vehicle touches the boundary line, as Figure 5(a) shows. The LPV controlled vehicle provides appropriate vehicle dynamics, while the uncontrolled vehicle displays irregular dynamics. The critical points are at stations $150 \mathrm{~m}$ and $175 \mathrm{~m}$. The uncontrolled vehicle fails during the test, while the LPV control is able to guarantee the avoidance of lane departure. Furthermore, Figure 5(b) shows the lateral distance of the vehicle from the boundary line. It can be stated that LPV control is able to guarantee the appropriate distance from the boundaries along the entire trajectory even at the critical sections, while the uncontrolled vehicle fails at the test.

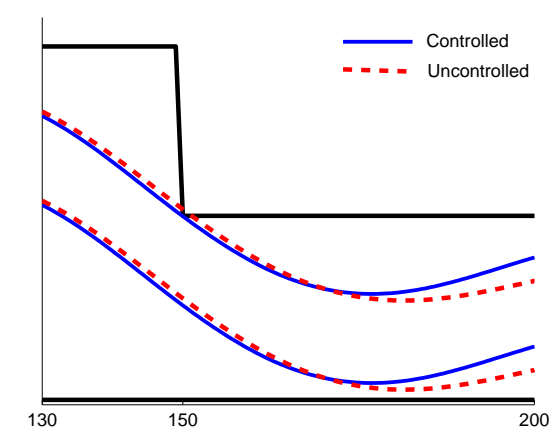

(a) Paths of the LPV and the uncontrolled vehicles

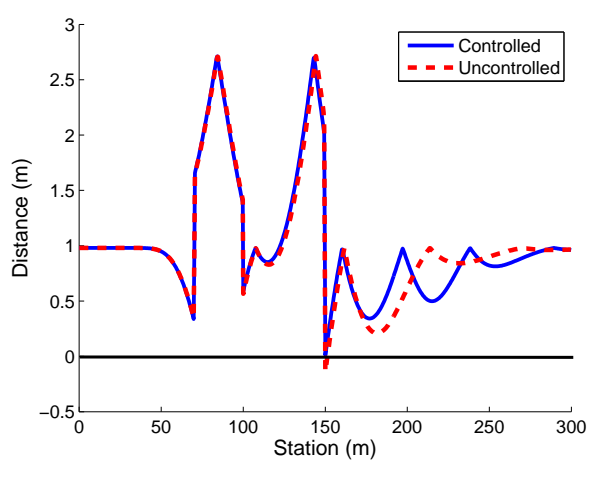

(b) Lateral distance from the borders

Figure 5. Trajectory tracking of the vehicle

Further signals of the simulation scenario are shown in Figure 6. Figure 6(a) illustrates the control input camber angle. The significant actuation between $150 \mathrm{~m} \ldots 250 \mathrm{~m}$ results in the improvement of vehicle dynamics. The scheduling variables of the control are depicted in Figure 6(b). The values of $\rho$ show that the vehicle moves in the nonlinear tire region, in which the proposed LPV control is also efficient. 


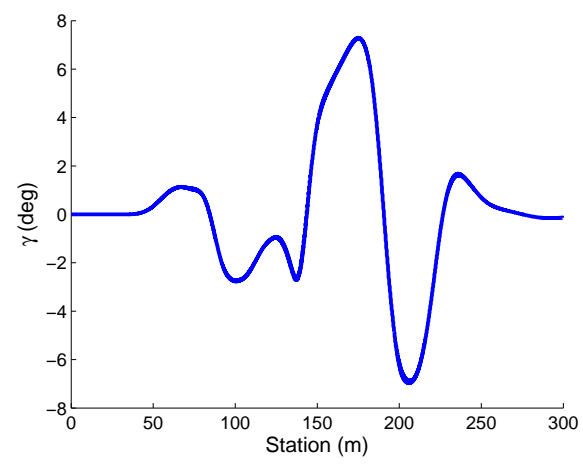

(a) Control input

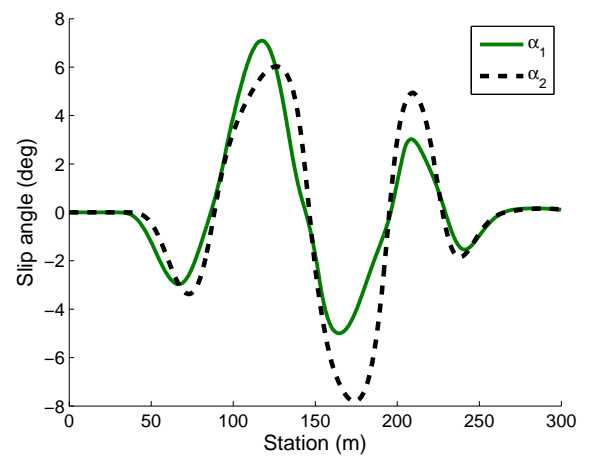

(b) Tyre slip angles

Figure 6. Simulation results of the maneuver

\section{CONCLUSIONS}

In the paper a method for the analysis and control design of variable-geometry suspension has been proposed. Both analysis and control methods handle the nonlinearities of the tire characteristics, which provides an extended application possibility for the vehicle dynamical actuator. The actuation of the system is controlled through an LPV control, which guarantees the trajectory tracking of the vehicle. The efficiency of the control synthesis method is illustrated through simulation scenarios, in which the critical maneuvers are handled.

\section{ACKNOWLEDGEMENT}

This paper was supported by the János Bolyai Research Scholarship of the Hungarian Academy of Sciences.

\section{REFERENCES}

Bokor, J. \& Balas, G. 2005. Linear parameter varying systems: A geometric theory and applications. 16th IFAC World Congress, Prague.

de Wit, C. C. \& Olsson, H. \& Astrom, K. J. \& Lischinsky, P. 1995. A new model for control of systems with friction. IEEE Trans. Automatic Control, 40(3):419-425.

Evers, W. \& van der Knaap, A. \& Besselink, I. \& Nijmeijer, H. 2008. Analysis of a variable geometry active suspension. Int. Symp. Advanced Vehicle Control, Kobe, Japan, pages 350-355.

Fallah, M. S. \& Bhat, R. \& Xie, W. F. 2009. New model and simulation of macpherson suspension system for ride control applications. Vehicle System Dynamics, 47(2):195-220.

Goodarzia, A. \& Oloomia, E. \& Esmailzadehb, E. 2010. Design and analysis of an intelligent controller for active geometry suspension systems. Vehicle System Dynamics, 49(1):333-359.

Kiencke, U. \& Nielsen, L. 2000. Automotive control systems for engine, driveline and vehicle. Springer.

Lee, S. \& Sung, H. \& Kim, J. \& Lee, U. 2006. Enhancement of vehicle stability 454 by active geometry control suspension system. International Journal of Automotive Technology, 7(3):303-307.

Lee, U. K. \& Lee, S. H. \& Han, C. S. \& Hedrick, K. \& Catala, A. 2008. Active geometry control suspension system for the enhancement of vehicle stability. Proc. IMechE, Part D: J. Automobile Engineering, 222(6):979-988.

Németh, B. \& Gáspár, P. 2012. Control design based on the integration of steering and suspension systems. IEEE Multiconference on Systems and Control. Dubrovnik, Croatia, pages 382-387.

Németh, B. \& Gáspár, P. 2012. Mechanical analysis and control design of mcpherson suspension. Int. J. Vehicle Systems Modelling and Testing, 7:173 - 193.

Németh, B. \& Gáspár, P. 2014. Set-based analysis of variable geometry suspension and steering system integration. 19th IFAC World Congress, pages 11201-11206.

Pacejka, H. B. 2004. Tyre and vehicle dynamics. Elsevier Butterworth-Heinemann, Oxford.

Piyabongkarn, D. \& Keviczky, T. \& Rajamani, R. 2004. Active direct tilt control for stability enhancement of a narrow commuter vehicle. International Journal of Automotive Technology, 5(2):77-88. 
Suarez, L. 2012. Active Tilt and Steer Control for a Narrow Tilting Vehicle: Control design and implementation. Lambert Academic Publishing.

Wu, F. \& Yang, X. H. \& Packard, A. \& Becker, G. 1996. Induced $l^{2}$-norm control for LPV systems with bounded parameter variation rates. International Journal of Nonlinear and Robust Control, 6:983-998. 